\title{
Increasing chlamydia test of re-infection rates using SMS reminders and incentives
}

\author{
Sandra Gaye Downing, ${ }^{1,2}$ Colette Cashman, ${ }^{1}$ Heather McNamee, ${ }^{1}$ Debbie Penney, ${ }^{1}$ \\ Darren B Russell, ${ }^{1}$ Margaret E Hellard ${ }^{2}$
}

${ }^{1}$ Cairns Sexual Health Service, Queensland Health, Cairns, Queensland, Australia

${ }^{2}$ Centre for Population Health, Burnet Institute, Melbourne, Victoria, Australia

\section{Correspondence to}

Sandra Gaye Downing, Cairns Sexual Health Service, PO Box 902, Cairns 4870, Queensland, Australia; sandra_downing@ health.qld.gov.au

Accepted 26 May 2012 Published Online First 23 June 2012

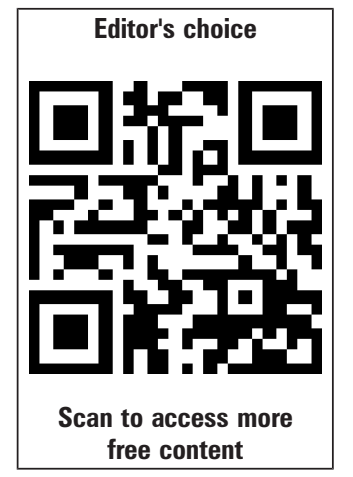

\begin{abstract}
Background Clients diagnosed and treated for Chlamydia trachomatis are a recognised high-risk group for subsequent infection. An estimated 8\% of clients treated for chlamydia at Cairns Sexual Health Service return for re-testing within the recommended 3-4-month period. There is no recall or reminder system in place. This study assesses the effectiveness of using short messaging service (SMS) reminders with and without incentive payments to increase re-testing rates.

Methods Eligible consenting clients were randomly allocated to one of three groups. Group 1 (controls) received the standard advice from the clinician to return for re-testing in 3-4 months. Group 2 received the standard advice and an SMS reminder at 10-12 weeks post-treatment. Group 3 received the standard advice and the SMS reminder, which also offered an incentive payment on clinic attendance.
\end{abstract}

Results 32 participants were recruited to groups 1 and 2 and 30 participants to group 3. 62 SMS reminders were sent with $13(21.0 \%)$ reported as undelivered. Re-testing rates were $6.3 \%, 28.1 \%$ and $26.7 \%$ for groups 1, 2 and 3, respectively.

Conclusion SMS reminders with or without an incentive payment increased re-testing rates in our clients who were diagnosed and treated for chlamydia. However, re-testing remained less than ideal, and the high rate of undelivered SMS reminders suggest that this intervention alone will not achieve desired re-testing rates and that a range of strategies will be required to increase re-testing in this population.

\section{INTRODUCTION}

Chlamydia trachomatis is the most frequently notified infection in Australia, with 74305 newly diagnosed infections reported in $2010 .^{1}$ The population rate of reported diagnoses in both men and women has more than tripled over the past 10 years. ${ }^{1}$ Untreated infections can have serious sequelae, most notably pelvic inflammatory disease in women, which can lead to chronic pelvic pain, ectopic pregnancy and infertility. ${ }^{2}$ Re-infection with chlamydia increases the risk for pelvic inflammatory disease and ectopic pregnancy in women ${ }^{3}$ and has been associated with increased risk of HIV seroconversion in men who have sex with men. ${ }^{4}$

Previous chlamydial infection as a predictor of subsequent infection in both men and women has been well documented ${ }^{5-8}$ and supports Australian clinical guideline recommendations of re-testing
3 months after a chlamydia diagnosis. ${ }^{9} 10$ There is a paucity of literature documenting re-testing rates; however, a recent paper from the Australian Collaboration for Chlamydia Enhanced Sentinel Surveillance (ACCESS) reports an overall 14.1\% re-testing rate across a 4-year period from the sexual health service network. ${ }^{11}$ The ACCESS Family Planning Network reported a $12.7 \%$ re-testing rate in individuals aged 16-29 years during 2008 and 2009. ${ }^{12}$ Cairns Sexual Health Service (CSHS) clients diagnosed with chlamydia are advised by a clinician at the time of treatment to return for re-testing in 3-4 months; however, CSHS has no recall or reminder system in place.

Short messaging service (SMS), also known as text messaging, transmits messages to mobile telephones and is an effective method of communicating appointment reminders and reducing 'did not attend' rates in outpatient settings. ${ }^{13}{ }^{14}$ Financial incentives have been shown to increase patients' compliance with healthcare treatment. ${ }^{15}$ The only study documenting the use of financial incentives to encourage clients to return for chlamydia re-testing showed little effect. ${ }^{16}$ However, the lack of effect of incentives in this study may result from the long time period from time of enrolment when incentives were discussed and the re-testing visit. In this study, we assessed the effectiveness of using SMS reminders with and without a financial incentive to increase re-testing rates in clients diagnosed with chlamydia at the CSHS.

\section{METHODS}

Clients attending the CSHS for treatment of chlamydia or who presented with genital symptoms (genital ulceration, vaginal discharge, abnormal vaginal bleeding, pelvic pain, dysuria) or who were a contact of someone diagnosed with chlamydia and who were aged at least 16 years, were residing in Cairns for the next 6 months, had access to a mobile telephone and were willing to receive an SMS reminder for a chlamydia test were invited to participate. HIV-positive clients were excluded as their regular HIV clinic appointments could influence re-testing patterns.

After recruitment, the chief investigator consecutively allocated study participants to a randomised list of numbers 1-3, generated using Excel software. Neither the clinicians recruiting the participants nor the participants themselves were informed of the randomisation outcome. Participants in group 1 (controls) received the standard 
advice from clinicians to return for re-testing in 3-4 months. Group 2 participants received the standard advice and a SMS reminder at 10-12 weeks post-treatment, and group 3 participants received the standard advice and an SMS reminder at 10-12 weeks post-treatment, which also offered a $\$ 10$ incentive payment on return to the clinic. Clients recruited but subsequently found not to have a chlamydial infection were withdrawn from the study.

A clinic mobile telephone was set up with the SMS reminder templates ' 3 mths $r$ up, drop in 4 a checkup or call 40506205 for an appointment' and ' $3 \mathrm{mths} r$ up, drop in 4 a check-up or call 40506205 for an appt \& get $\$ 10$ '. SMS reminders were manually sent on Mondays, or Tuesdays if Monday was a public holiday, for all participants due for a reminder in the coming week. Delivery reports were received. The messaging system would intermittently attempt to deliver an SMS reminder for up to 7 days before it was reported as 'undelivered'.

Participants had a range of options for re-testing-a booked appointment or a walk-in appointment where they were reviewed by a member of nursing staff or they could simply provide a urine sample (arranged by the reception staff) without a formal clinical review. Participants were considered 're-tested' if they had a chlamydia test between 10 and 16 weeks post-treatment.

During 2008, CSHS diagnosed approximately 120 chlamydia infections in clients residing in the Cairns area, with an estimated 8\% re-testing within 3-4 months. Sample size calculations were made based on this information. To detect a $10 \%$ absolute difference in re-testing rates between group 1 and group 2 , and between group 1 and group 3, 30 participants were required in each group (power $=80 \%, \dot{\alpha}=5 \%$ ).

The $\chi^{2}$ test was used to compare characteristics of participants in each group and to assess associations with an undelivered SMS reminder. We calculated the proportion of participants in each group re-tested and used a $\chi^{2}$ test (twosided Fisher's exact) to determine if there was a significant difference in re-testing rates. Both an intention-to-treat analysis and a per-protocol analysis were conducted. Stata statistical software (V.10) was used to conduct all analyses.

Ethical approval for this study was obtained through the Cairns and Hinterland Health Service District Human Research Ethics Committee.

\section{RESULTS}

Between January 2010 and March 2011, 161 clients were invited to participant in the study, of whom 94 were identified as having chlamydia infection and were followed throughout the re-testing period-32 participants in groups 1 and 2 and 30 participants in group 3 .

Fifty-nine (62.8\%) participants were aged $<25$ years, $28.7 \%$ identified as being Aboriginal and/or Torres Strait Islander and there was a similar proportion of men and women. There was no statistically significant difference in characteristics between the three groups (table 1).

Sixty-two SMS reminders were sent with $13(21.0 \%)$ reported as undelivered. Seven of the 13 participants (53.8\%) with undelivered SMS reminders identified as Aboriginal and/or Torres Strait Islander. This association was statistically significant $(p=0.047)$. There was no association between sex or age group and undelivered SMS reminders.

Chlamydia re-testing rates among group 1 (controls) were $6.3 \%$, and using an intention-to-treat analysis, group 2 (SMS reminders) had a re-testing rate of $28.1 \%$ and group 3 (SMS reminders and incentive) a $26.7 \%$ re-testing rate (table 2 ). Of the
Table 1 Participant characteristics by study group

\begin{tabular}{|c|c|c|c|c|c|}
\hline & $\begin{array}{l}\text { All } \\
(\mathrm{n}=94) \\
\mathrm{n}(\%)\end{array}$ & $\begin{array}{l}\text { Group 1 } \\
(\mathrm{n}=32) \\
\mathrm{n}(\%)\end{array}$ & $\begin{array}{l}\text { Group 2 } \\
(\mathrm{n}=32) \\
\mathrm{n}(\%)\end{array}$ & $\begin{array}{l}\text { Group 3 } \\
(\mathrm{n}=30) \\
\mathrm{n}(\%)\end{array}$ & p Value \\
\hline \multicolumn{6}{|l|}{ Sex } \\
\hline Male & $46(48.9)$ & $14(43.8)$ & $16(50.0)$ & $16(53.3)$ & 0.744 \\
\hline Female & $48(51.1)$ & $18(56.3)$ & $16(50.0)$ & $14(46.7)$ & \\
\hline \multicolumn{6}{|c|}{ Age group (years) } \\
\hline$<25$ & $59(62.8)$ & $20(62.5)$ & $18(56.3)$ & $21(70.0)$ & 0.534 \\
\hline$\geq 25$ & $35(37.2)$ & $12(37.5)$ & $14(43.8)$ & $9(30.0)$ & \\
\hline \multicolumn{6}{|c|}{ Indigenous status } \\
\hline ATSI & 27 (28.7) & $10(31.3)$ & $9(28.1)$ & $8(26.7)$ & 0.387 \\
\hline Non-ATSI & $61(64.9)$ & $20(62.5)$ & 19 (59.4) & $22(73.3)$ & \\
\hline Not stated & $6(6.4)$ & $2(6.3)$ & $4(12.5)$ & $0(0.0)$ & \\
\hline
\end{tabular}

19 participants who re-tested, 3 (15.9\%) were found to have a chlamydial infection. There was a significant difference in re-testing rates between group 1 and both group $2(p=0.043)$ and group $3(p=0.040)$ in this analysis.

In the per-protocol analysis with exclusions of those who did not re-test within the defined 10-16 week post-treatment period and those with an undelivered SMS reminder or who re-tested for chlamydia prior to being sent an SMS reminder, none of group 1 participants re-tested, 5 (22.7\%) of group 2 and seven $(29.2 \%)$ of group 3 participants were re-tested (table 3 , figure 1). Of these 12 participants who re-tested, two (16.6\%) had a chlamydial infection. There was a significant difference in re-testing rates between group 1 and both group $2(p=0.010)$ and group $3(p=0.002)$ in this analysis.

\section{DISCUSSION}

This is the first Australian study assessing the effectiveness of SMS reminders with and without incentive payments to increase re-testing rates following a chlamydia diagnosis. SMS reminders with or without an incentive payment increased re-testing rates when compared to no SMS reminder. Another notable finding is that despite the success of the SMS intervention, $21 \%$ of SMS reminders were undelivered.

SMS reminders with and without an incentive payment significantly increased chlamydia re-testing rates to $26.7 \%$ and $28.1 \%$, respectively. Various other strategies to increase re-testing rates after a chlamydia diagnosis have been trialled, also with moderate success. ${ }^{16-18}$ Paneth-Pollak et al ${ }^{18}$ found that the use of postcard reminders increased re-testing rates from $7.7 \%$ to $14.1 \%$. Malotte et al $^{16}$ trialled a number of strategies concluding that a telephone call was the most effective method and similar to our study that monetary incentives did not increase re-testing rates compared with a brief recommendation. Despite the seeming lack of benefit from small incentive payments being provided with SMS influencing re-testing rates compared with SMS alone, this result must be viewed with caution due to the

Table 2 Comparison of chlamydia re-testing by study group (intentionto-treat analysis)

\begin{tabular}{|c|c|c|c|c|}
\hline & $\begin{array}{l}\text { Not } \\
\text { re-tested } \\
\text { n }\end{array}$ & $\begin{array}{l}\text { Re-tested } \\
\text { n }\end{array}$ & $\begin{array}{l}\text { Re-tested } \\
\%(95 \% \mathrm{CI})\end{array}$ & p Value \\
\hline Group 1 controls ( $n=32$ ) & 30 & 2 & 6.3 (0.8 to 20.8 ) & \\
\hline $\begin{array}{l}\text { Group } 2 \text { SMS reminder } \\
(\mathrm{n}=32)\end{array}$ & 23 & 9 & 28.1 (13.8 to 46.6$)$ & 0.043 \\
\hline $\begin{array}{l}\text { Group } 3 \text { SMS reminder } \\
\text { and incentive }(n=30)\end{array}$ & 22 & 8 & 26.67 (12.3 to 45.9$)$ & 0.040 \\
\hline
\end{tabular}


Table 3 Comparison of chlamydia re-testing by study group (perprotocol analysis)

\begin{tabular}{lllll}
\hline & $\begin{array}{l}\text { Not } \\
\text { re-tested } \\
\mathbf{n}\end{array}$ & $\begin{array}{l}\text { Re-tested } \\
\mathbf{n}\end{array}$ & $\begin{array}{l}\text { Re-tested } \\
\%(\mathbf{9 5 \%} \mathbf{C l})\end{array}$ & p Value \\
\hline $\begin{array}{l}\text { Group 1 controls }(\mathrm{n}=30) \\
\begin{array}{l}\text { Group 2 SMS reminder } \\
(\mathrm{n}=22)\end{array}\end{array}$ & 17 & 0 & 0 & \\
$\begin{array}{l}\text { Group 3 SMS reminder } \\
\text { and incentive ( } \mathrm{n}=24)\end{array}$ & 17 & 5 & $22.7(8.8$ to 43.4) & 0.010 \\
\hline
\end{tabular}

small sample size in our study. The highest re-testing rates were reported by Sparks et al ${ }^{17}$ where clients were contacted 10 weeks after treatment and offered the option of returning to the clinic or being sent a postal specimen collection kit to mail back. However, this was a small study and involved a personal telephone call to enrol participants, an intervention within itself. Importantly, $42 \%$ of potentially eligible participants were unable to be contacted for possible enrolment.

A study measuring re-testing across Australia's sexual health services (the ACCESS study network) between 2004 and 2008 showed no significant increasing trend in re-testing suggesting that any interventions to increase re-testing have had minimal success. ${ }^{11}$ A wide range of re-testing rates, $5.7 \%-32.0 \%$, was reported across the 19 reporting clinics. A more in-depth analysis is required to determine if this variation is a reflection of the different client populations accessing these clinics or specific re-testing interventions. The ACCESS analysis included the entire clinic population, whereas our study had specific inclusion and exclusion criteria making direct comparison problematic.

An important finding from this study is that $21.0 \%$ of SMS reminders were undelivered despite the mobile telephone numbers being current at the time of enrolment 10-12 weeks prior. If the recipient's mobile telephone is turned off, out of range or has a full-text message inbox, the message may not be received immediately. However, as the messaging system attempted to deliver the SMS reminder for a period of time before reporting it as undelivered, it is more likely that the recipient's telephone had been deactivated or suspended. Reasons for deactivation or suspension of a mobile telephone include non-payment of a post-paid service, the telephone being reported as lost or stolen, transfer of the number to a different service provider or not recharging within a defined time period.

Despite small numbers in this study, there was an association between an undelivered SMS reminder and clients identifying as Aboriginal and/or Torres Strait Islander. The reasons for this are unclear; however, it is particularly important given Aboriginal

Figure 1 Flow of participants.

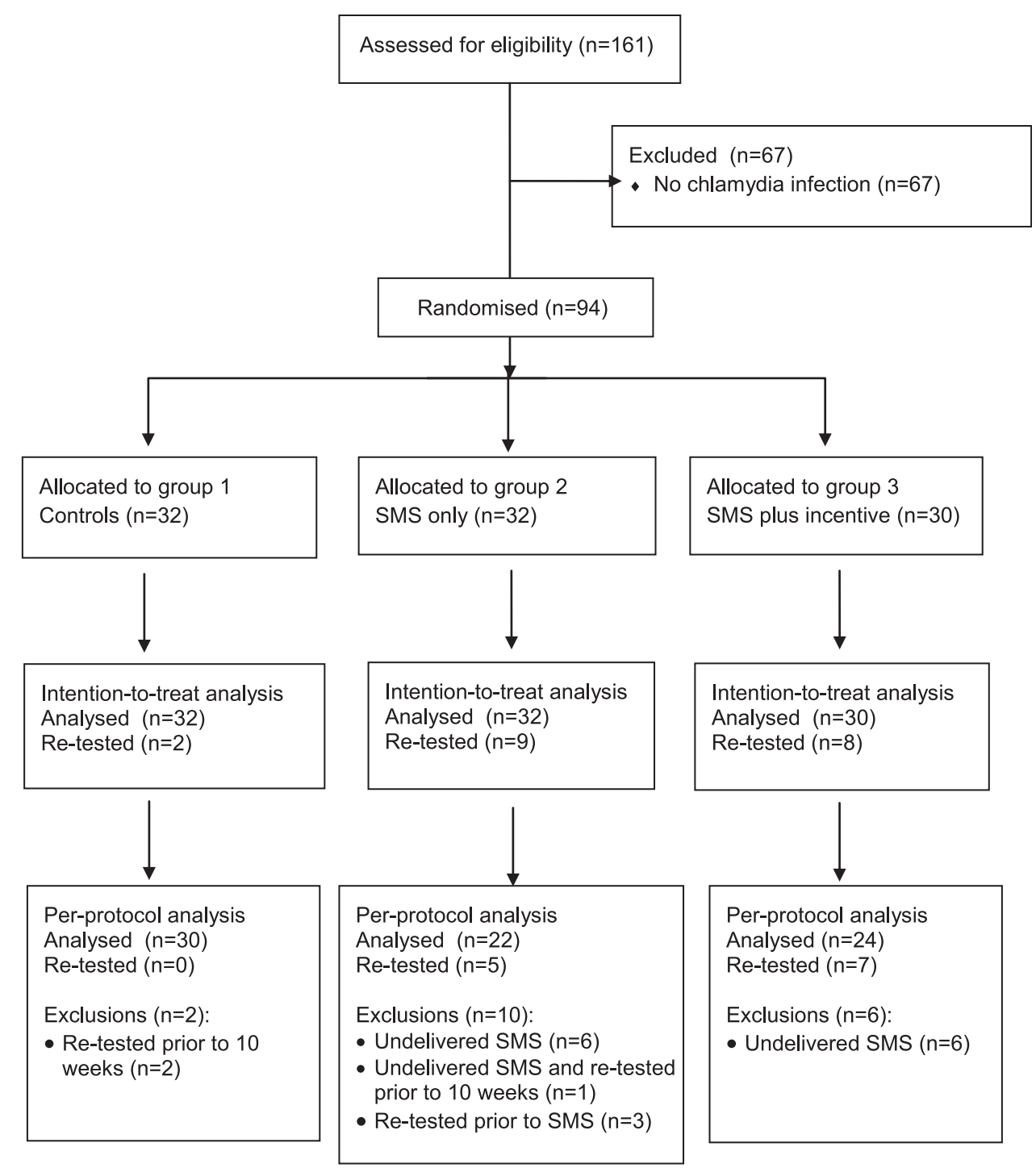




\section{Key messages}

- SMS reminders with incentive payments significantly increased re-testing rates; however, rates remained less than ideal.

- A high proportion of SMS reminders were 'undelivered', and effectiveness of this intervention in different population groups should be evaluated.

and Torres Strait Islander people are a known high-risk group for chlamydia infection. A greater understanding of mobile telephone usage in key population groups is required to determine if this intervention would be suitable for re-testing reminders.

Despite the relative success of this intervention, neither intervention group achieved a re-testing rate $>28.1 \%$. In a nonstudy environment, this rate could be even lower as clinicians may not place the same emphasis on re-testing as they might have during this study. Additional systems for recall such as telephone, email or postcard reminders and options for mail-out testing kits where clinic re-attendance is not required may improve re-testing rates. Offering a range of reminder and re-testing alternatives would enable clients to determine the option best suited to their circumstances and should be considered. Motivational interviewing and greater emphasis on the importance of re-testing by the clinician at the time of treatment should also be further investigated.

This study has some limitations. A larger sample size would have enabled analysis of associations between participant characteristics and between group 2 (SMS reminder) and group 3 (SMS reminder plus incentive) re-testing rates. Although participant characteristics across the three groups were broadly similar, the small sample size and numerical differences between the groups could have influenced the results. Additionally, the CSHS relocated during the study period, and this may have influenced whether participants returned for re-testing or not. Finally, the high proportion of undelivered SMS messages was unexpected.

Recall/reminder systems using SMS technology provide a simple effective method to target high-risk groups for re-testing and should be considered an integral tool in modern health services. A larger study to determine the impact incentive payments in combination with SMS reminders have on re-testing rates would be valuable in guiding future strategies. However, the high rate of undelivered SMS reminders indicates that this intervention alone will not achieve desired re-testing rates and its effectiveness in different population groups should be evaluated. The less than optimal re-testing rates achieved in previous studies also suggest that a combination of innovative interventions will be required to increase re-testing in this population.

Acknowledgements We thank the staff of Cairns Sexual Health Service for their enthusiasm and commitment to this study, Tim Spelman from the Burnet Institute for statistical advice and the study participants. Margaret Hellard is supported by an NHMRC Senior Research Fellowship.

Contributors SGD: (1) substantial contribution to conception and design and analysis and interpretation of data, (2) drafting the article and (3) final approval of the version to be published. CC: (1) acquisition of data, (2) revising the article critically for important intellectual content and (3) final approval of the version to be published.
HM: (1) acquisition of data, (2) revising the article critically for important intellectual content and (3) final approval of the version to be published. DP: (1) acquisition of data, (2) revising the article critically for important intellectual content and (3) final approval of the version to be published. DBR: (1) substantial contribution to conception and design and acquisition of data, (2) revising the article critically for important intellectual content and (3) final approval of the version to be published. $\mathrm{MH}$ : (1) substantial contribution to conception and design and analysis and interpretation of data, (2) revising the article critically for important intellectual content and (3) final approval of the version to be published.

Funding The Queensland Nursing Council provided funding for this study.

\section{Competing interests None.}

Patient consent Obtained.

Ethics approval Ethics approval was provided by Cairns and Hinterland Health Research Ethics Committee.

Provenance and peer review Not commissioned; externally peer reviewed.

Data sharing statement The data are held at Cairns Sexual Health Service, and all authors have access to them through the corresponding author.

\section{REFERENCES}

1. The Kirby Institute. HIV Viral Hepatitis and Sexually Transmitted Infection in Australia Annual Surveillance Report 2011. Sydney, NSW: The Kirby Institute, the University of New South Wales, 2011.

2. Low $\mathbf{N}$, Egger $\mathrm{M}$, Sterne $\mathrm{JA}$, et al. Incidence of severe reproductive tract complications associated with diagnosed genital chlamydial infection: the Uppsala Women's Cohort Study. Sex Transm Infect 2006:82:212-18.

3. Hillis SD, Owens LM, Marchbanks PA, et al. Recurrent chlamydial infections increase the risks of hospitalization for ectopic pregnancy and pelvic inflammatory disease. Am J Obstet Gynecol 1997;176:103-7.

4. Berstein KT, Marcus JL, Nieri G, et al. Rectal gonorrhoea and chlamydia reinfection is associated with increased risk of HIV seroconversion. J Acquir Immune Defic Syndr 2010;53:537-43

5. Dunne EF, Chapin JB, Rietmeijer CA, et al. Rate and predictors of repeat Chlamydia trachomatis infection among men. Sex Transm Dis 2008;35(11 Suppl):S40-4.

6. Rietmeijer CA, Van Bemmelen R, Judson FN, et al. Incidence and repeat infection rates of Chlamydia trachomatis among male and female patients in an STD clinic: implications for screening and rescreening. Sex Transm Dis 2002;29:65-72.

7. Scott Lamontagne D, Baster K, Emmett L, et al. Incidence and reinfection rates of genital chlamydial infection among women aged 16-24 years attending general practice, family planning and genitourinary medicine clinics in England: a prospective cohort study by the Chlamydia Recall Study Advisory Group. Sex Transm Infect 2007; 83:292-303.

8. Whittington WL, Kent $C$, Kissinger $P$, et al. Determinants of persistent and recurrent Chlamydia trachomatis infection in young women: results of a multicenter cohort study. Sex Transm Dis 2001;28:117-23.

9. Australian Chapter of Sexual Health Medicine. National Management Guidelines for Sexually Transmissible Infections. Melbourne: Royal Australasian College of Physicians, 2008.

10. Sexually Transmissible Infection in Gay Men Action Group. Sexually Transmitted Infection Testing Guidelines for Men Who Have Sex With Men (STIGMA Guidelines). Sydney: Australasian Chapter of Sexual Health Medicine, 2008.

11. Guy R, Wand $\mathrm{H}$, Franklin $\mathrm{N}$, et al. Re-testing for chlamydia at sexual health services in Australia, 2004-08. Sex Health 2011:8:242-7.

12. Bowring A, Gouillou M, Guy R, et al. Chlamydia Repeat Testing and Positivity Rates at General Practices and Family Planning Clinics in Australia 2008-2009. Canberra ACT, Australia: Australasian Sexual Health Conference, 2011.

13. Geraghty $\mathbf{M}$, Glynn $\mathbf{F}, A \min \mathbf{M}$, et al. Patient mobile telephone 'text' reminder: a novel way to reduce non-attendance at the ENT out-patient clinic. J Laryngol Otol 2008;122:296-8.

14. Milne RG, Horne M, Torsney B. SMS reminders in the UK national health service: an evaluation of its impact on "no-shows" at hospital out-patient clinics. Health Care Manage Rev 2006;31:130-6.

15. Giuffrida A, Torgerson DJ. Should we pay the patient? Review of financial incentives to enhance patient compliance. BMJ 1997;315:703-7.

16. Malotte CK, Ledsky $\mathrm{R}$, Hogben $\mathrm{M}$, et al. Comparison of methods to increase repeat testing in persons treated for gonorrhea and/or chlamydia at public sexually transmitted disease clinics. Sex Transm Dis 2004;31:637-42.

17. Sparks R, Helmers JR, Handsfield HH, et al. Rescreening for gonorrhea and chlamydial infection through the mail: a randomized trial. Sex Transm Dis 2004;31:113-16

18. Paneth-Pollak R, Klingler EJ, Blank S, et al. The elephant never forgets; piloting a chlamydia and gonorrhea retesting reminder postcard in an STD clinic setting. Sex Transm Dis 2010:37:365-8. 